\title{
Demonstration of interactive teaching for end-to-end dialog control with hybrid code networks
}

\author{
Jason D. Williams \\ Microsoft Research \\ jason.williams@microsoft.com
}

\author{
Lars Liden \\ Microsoft \\ lars.liden@microsoft.edu
}

\begin{abstract}
This is a demonstration of interactive teaching for practical end-to-end dialog systems driven by a recurrent neural network. In this approach, a developer teaches the network by interacting with the system and providing on-the-spot corrections. Once a system is deployed, a developer can also correct mistakes in logged dialogs. This demonstration shows both of these teaching methods applied to dialog systems in three domains: pizza ordering, restaurant information, and weather forecasts.
\end{abstract}

\section{Introduction}

Whereas traditional dialog systems consist of a pipeline of components such as intent detection, state tracking, and action selection, an end-to-end dialog system is driven by a machine learning model which takes observable dialog history as input, and directly outputs a distribution over dialog actions. The benefit of this approach is that intermediate quantities such as intent or dialog state do not need to be labeled - rather, learning can be done directly on example dialogs.

In practice, purely end-to-end methods can require large amounts of data to learn seemingly simple behaviors, such as sorting database results. This is problematic because when building a new dialog system, typically no in-domain dialog data exists, so data efficiency is crucial. Moreover, machine-learned models alone cannot guarantee practical constraints are followed - for example a bank would require that a user must be logged in before they are allowed to transfer funds. For these reasons, in past work we introduced Hybrid Code Networks (HCN) (Williams et al., 2017). HCNs make end-to-end learning of task-oriented dialog systems practical by combining a recurrent neural network (RNN) with domain-specific software provided by the developer; domain-specific action templates; and a conventional entity extraction module for identifying entity mentions in text. Experiments on a public corpus show that HCNs can substantially reduce the number of training dialogs required compared to purely end-to-end learning methods, and also outperform purely rule-based systems.

This demonstration shows a practical implementation of HCNs, as a web service for building task-oriented dialog systems. Once the developer has provided their domain-specific software, they can add training dialogs in several ways. First, the developer can simply upload dialogs to the training set. Second, the developer can interactively teach the $\mathrm{HCN}$, and make on-the-spot corrections. Finally, as the HCN interacts with end-users, the developer can inspect logged dialogs, make corrections if needed, and add the dialogs to the training set.

\section{Dialog learning platform}

The practical operation of the HCN is shown in Figure 1, where the left-hand block in white shows an end-user messaging client, the center block in blue shows a web service implemented by the system developer that hosts domain-specific logic, and the right-hand block in green is the HCN web service. A software development kit (SDK) facilitates using the HCN web service.

When interacting with end users, the process begins when the end user provides input text, such as "What's the 5 day forecast for Seattle?", shown as item 1 in Figure 1. This text can be typed or output by a standard speech recognizer. This text is passed to the developer's web service, which in turn calls the HCN service to perform entity ex- 


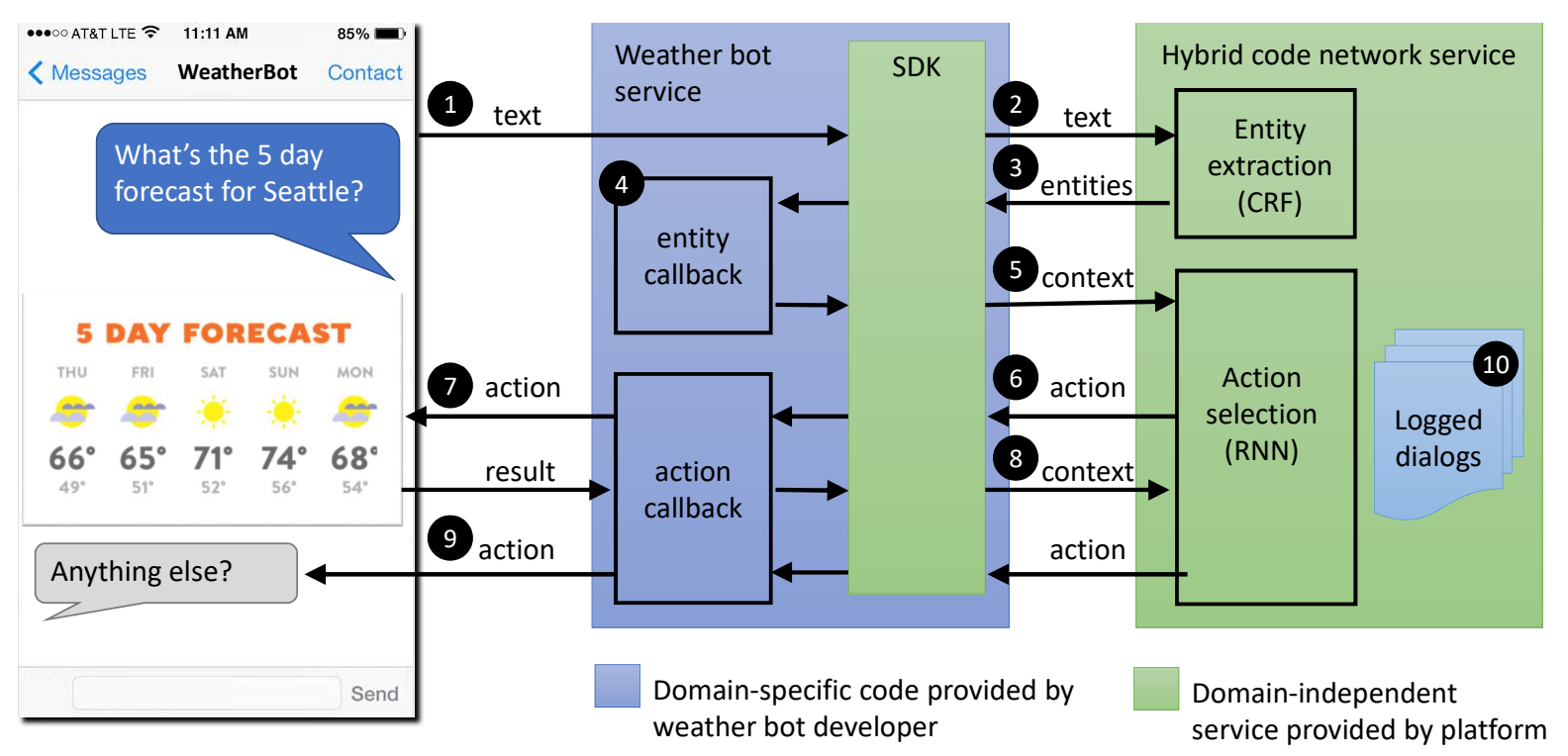

Figure 1: Development platform for interactive dialog learning. Entity extraction is done with Conditional Random Fields (CRFs). See text for full details.

traction (item 2). The HCN service then returns entity mentions detected in text, such as "location=Seattle" (3). Domain-specific code on the developer's service then resolves entity mentions to a canonical form, such as a latitude/longitude pair, and to store entities for use in later turns in the dialog (4). The developer's code then calls the HCN service again, optionally passing in context which can include which entities have been recognized so far in the dialog, as well as an action mask that limits which action templates are available at the current step (5).

The HCN service returns a distribution over all un-masked action templates, and the developer code executes the highest-ranked action (6). If this action template is an API call - such as displaying rich content to the user, executing a transaction in a database, or raising a robot's arm - that API is invoked (7), and the HCN service is called again to choose the next action. If the API call returns context features, those can be passed to the HCN service (8). If the action template is text, the developer's code can substitute in entity values such as a weather forecast, and the text is rendered to the end user (9). The cycle then repeats.

Dialogs conducted with users are logged by the HCN service, and can later be reviewed and corrected by the system developer through a web user interface (10). Also, the cycle can be augmented to support interactive teaching. These aspects are described in the next section.

\section{Illustrative interactions}

When creating a new dialog system, typically no in-domain data exists. To address this, the dialog learning platform supports interactive teaching. In interactive teaching, the developer alternates between the role of the end user, and the role of the teacher. The operational loop shown in Figure 1 is modified so that results of entity extraction and action selection can be corrected before continuing.

Figure 4 shows an example of interactive teaching for pizza ordering. The developer - playing the part of the user - enters "medium pizza with olives". The current entity extraction model finds entity mentions for the \$pizza and \$size entities, but not the "olive" \$topping. So, the developer corrects this by adding a corrected entity label, and this corrected label is used going forward. The interface then displays the contents of the developerdefined state, and provides a list of actions, each with their score under the current RNN model. In this example, all but one of the actions are shown as "disqualified", meaning that the action mask prohibits them. For example, the action "Would you like a Small, Medium, or Large \$crust pizza ..." is masked because the pizza size is already known. The developer enters the index of the action to take ("1") and the dialog continues. At this point, the developer could have alternatively entered a new action - for example, by typing "So you want \$toppings, is that right?". As each correction is made, the CRF and RNN models are re- 


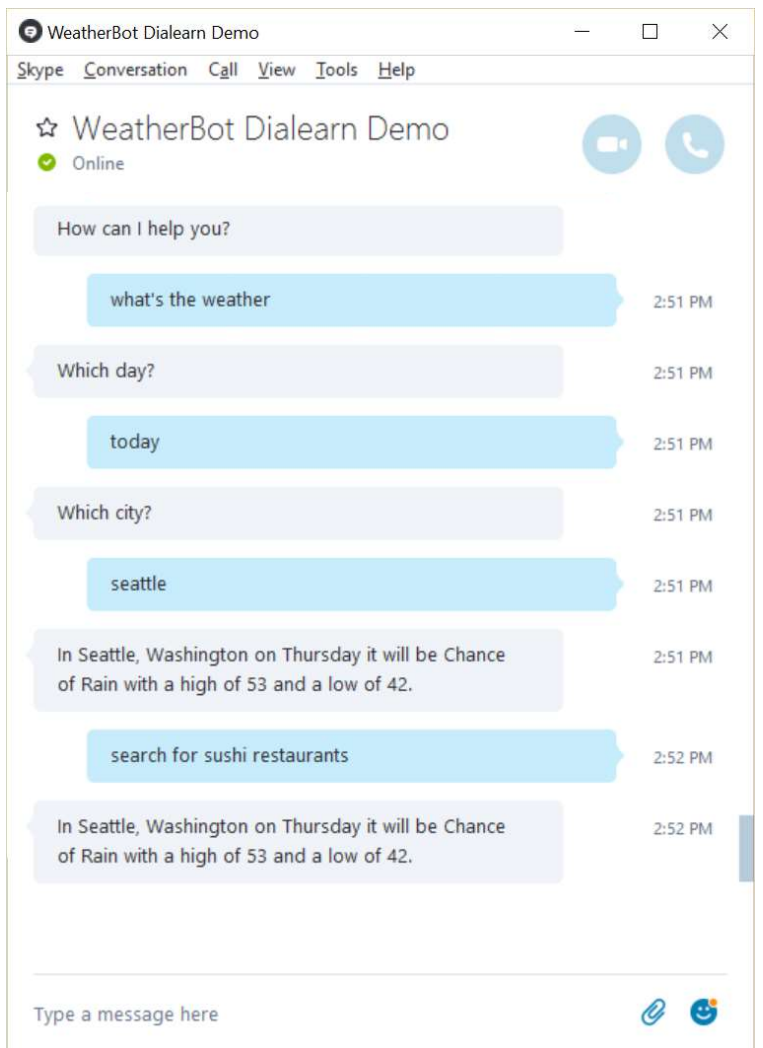

Figure 2: Example interaction with an end user. Note the system mistake after the user enters "search for sushi restaurants".

trained.

Once a rudimentary model is in place, endusers can start using the system. An example dia$\log$ with an end-user is shown in Figure 2, which shows an error at the last system turn. Figure 3 shows how this dialog appears to the developer, and how a correction can be made. Each system utterance is shown in a drop-down box. If the developer identifies a turn where the system output the wrong action, the developer can select the correct action from the drop-down. When an action which differs from the action in the log is selected, the remainder of the dialog is discarded, since it is no longer known how the user would have responded. If none of the actions is appropriate, the developer can choose "new action...", and enter a new action into a provided text box. When the dialog has been corrected, the developer clicks on "submit", which saves the labeled dialog to the training set, re-trains the model, and re-deploys the new model. In the example in Figure 3, the user's fourth input was "search for sushi

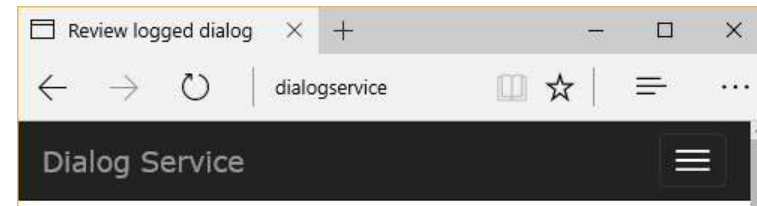

Applications $>$ Weather demo app $>$ logs

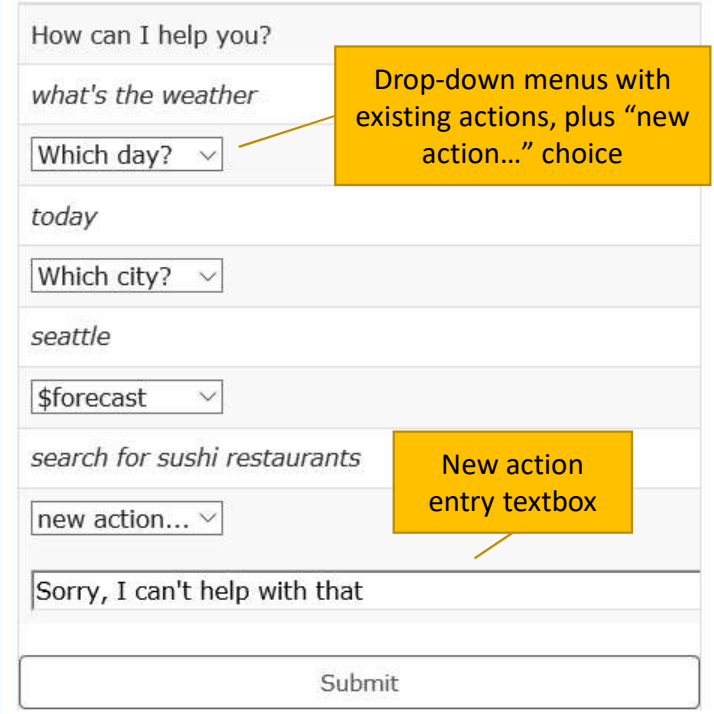

Figure 3: Example of off-line dialog correction, showing the dialog collected in Figure 2. After the user says "search for sushi restaurants", the developer changed the action "\$forecast" to "new action..." and typed in "Sorry, I can't help with that".

restaurants", and the system had answered with a weather forecast. The developer changed this response to "new action..." and typed in the new action "Sorry, I can't help with that".

In the demonstration, we have three working dialog systems available, for pizza ordering, restaurant information, and weather forecasts. The demonstration shows applying the two interactive methods described above to each of these three domains.

\section{References}

Jason D Williams, Kavosh Asadi, and Geoffrey Zweig. 2017. Hybrid code networks: practical and efficient end-to-end dialog control with supervised and reinforcement learning. In Proc ACL, Vancouver. 


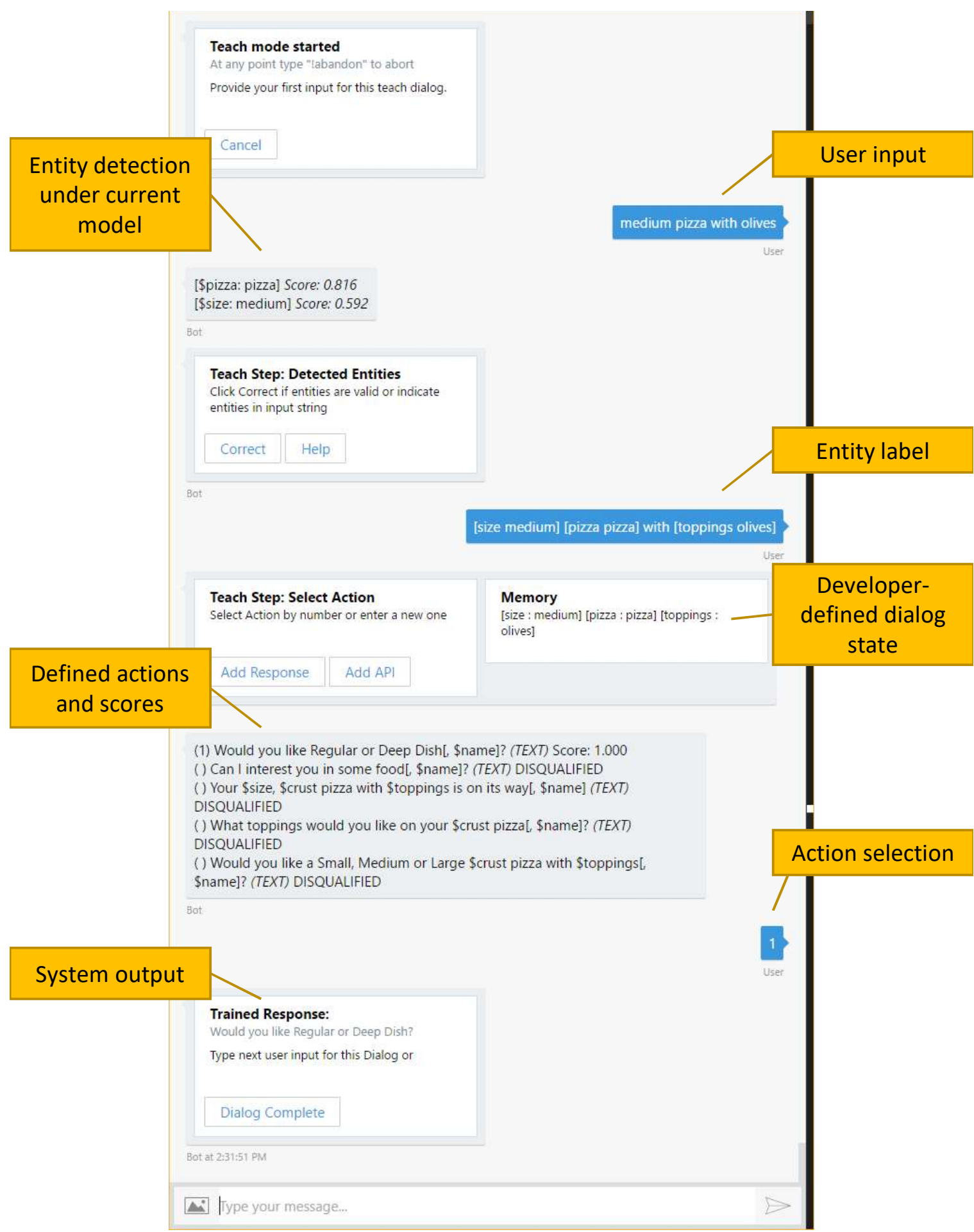

Figure 4: Example of interactive dialog teaching. The developer's input is in blue boxes on the right side, and the system's responses are in grey and white boxes on the left side. The developer alternates between playing the role of an end user, and providing corrective input. 